\title{
Narrow- and broadband Interference Robustness for OOK/BPPM based Energy Detection
}

\author{
Hanns-Ulrich Dehner, Holger Jäkel, and Friedrich K. Jondral \\ Karlsruhe Institute of Technology (KIT), Communications Engineering Lab, 76128 Karlsruhe, Germany \\ Email: \{hanns-ulrich.dehner, holger.jaekel, friedrich.jondral\}@kit.edu
}

\begin{abstract}
An analysis of narrow- and broadband interference robustness within an On-Off Keying/Binary Pulse Position Modulation based noncoherent multiband impulse radio ultrawideband communication system is presented. Using the energy detector's processing gain closed-form expressions of the noise and interference related second order moment statistics at the output of an energy detection receiver are derived. This allows separate statements on the relative modulation specific processing gain with respect to various interference parameters.
\end{abstract}

Index Terms-Energy detection, interference robustness, processing gain, UWB, OOK, BPPM.

\section{INTRODUCTION}

The fundamental resolutions of e.g. the Federal Communications Commission (FCC) [1] in 2002 or the Electronic Communications Committee (ECC) [2] in 2008 have lead to enormous research activities in academia and industry to enable a possible commercialization of the unlicensed ultrawideband (UWB) technology. Thereby, wireless community considers UWB as a possible candidate to revolutionize highspeed data transmissions as well as an enabler for the personal area networking industry aiming at novel innovations and a greater quality of the services to the end user.

As future UWB systems are required to be realized with low-complexity and in a power efficient way, a notable part of research focuses recently on suboptimal noncoherent UWB systems [3]. In contrast to coherent UWB systems, the main reasons to favor noncoherent UWB systems are

- the avoidance of a receiver-side high cost, high-speed and power consuming analog-to-digital converter,

- the relaxed synchronization constraints and

- the efficient handling of energy capture resulting from the high multipath diversity.

A promising noncoherent UWB system suited for high data rate transmissions is multiband impulse radio UWB (MIR UWB) [4], [5], [6], [7]. Therein, extremely short pulses are given to the input of an analog bandpass filterbank covering the available spectrum. The pulses at the filters' outputs are modulated with On-Off Keying (OOK)/Binary Pulse Position Modulation (BPPM), added up and afterwards transmitted. The receiver front-end uses the same bandpass filterbank to split up the received UWB signal followed by a parallelized energy detection.

A pivotal vulnerability of the energy detection receiver is its high sensitivity with respect to interference. Interference passing the filterbank might lead to a reduction of the instantaneous signal-to-interference-plus-noise ratio (SINR) and hence to a falsified decision process. For this reason, it is required to investigate the interference robustness of an energy detector regarding, e.g., different modulation schemes.

This paper bases on [8] which analyzes out-of-band interference for noncoherent UWB systems with BPPM based energy detection. Recently, [9] extends this approach to make investigations of in-band narrowband interference (NBI) for OOK/BPPM based energy detection. However, a general analytical investigation of narrow-and broadband interference robustness for energy detection of OOK/BPPM is not yet done to the best of the authors' knowledge.

The remainder of the paper is as follows: In Section II the signal model for OOK/BPPM based data transmission including the energy detection under interference is introduced. Section III defines the processing gain (PG) of the energy detector and analyzes the interference and noise related statistics at its output. The subsequent Section IV identifies the interference robustness for both modulation schemes using the energy detector's PG. Concluding remarks are given in Section $\mathrm{V}$.

\section{Signal Model}

\section{A. Transmitter}

A binary data transmission link within an MIR UWB subband of bandwidth $B$ is considered. Based on OOK/BPPM modulation the rectangular pulse

$$
p(t)= \begin{cases}\sqrt{\frac{2}{T_{\mathrm{p}}}} \cos \left(2 \pi f_{\mathrm{c}} t\right) & 0<t<T_{\mathrm{p}}, \\ 0 & \text { else, }\end{cases}
$$

with carrier frequency $f_{\mathrm{c}}$ and pulse duration $T_{\mathrm{p}}$ is emitted with energy $1\left(f_{\mathrm{c}} \gg 1 / T_{\mathrm{p}}\right)$. The resulting signal to be transmitted conducts to

$$
s_{\mathrm{O}}(t)=\sqrt{E_{\mathrm{p}}^{\mathrm{O}}} \sum_{k=-\infty}^{\infty} b_{k} p\left(t-k T_{\mathrm{b}}\right),
$$

for OOK and

$$
s_{\mathrm{P}}(t)=\sqrt{E_{\mathrm{p}}^{\mathrm{P}}} \sum_{k=-\infty}^{\infty} p\left(t-k T_{\mathrm{b}}-b_{k} \frac{T_{\mathrm{b}}}{2}\right),
$$

for BPPM. Data bit $b_{k} \in\{0,1\}$, which is assumed to be uniformly distributed, is specified by bit energy $E_{\mathrm{b}}$ as well as bit duration $T_{\mathrm{b}}=\frac{T_{\mathrm{p}}}{d_{\mathrm{s}}}$ with duty cycle $d_{\mathrm{s}} \leq \frac{1}{2}$. Finally, 
$E_{\mathrm{p}}^{i}, i \in\{\mathrm{O}, \mathrm{P}\}$ stands for the modulation specific pulse energy which equals $E_{\mathrm{p}}^{\mathrm{O}}=2 E_{\mathrm{p}}^{\mathrm{P}}=2 E_{\mathrm{b}}$.

\section{B. Receiver}

Assuming perfect synchronization between transmitter and receiver, the signal $s_{i}(t), i \in\{\mathrm{O}, \mathrm{P}\}$ is superposed with zero mean white Gaussian noise $n(t)$ of two-sided spectral density $\frac{N_{0}}{2}$ and interference $j(t)$ leading to (no fading)

$$
y(t)=s_{i}(t)+n(t)+j(t) .
$$

Interference is described as band-limited wide-sense stationary, time-continuous zero mean Gaussian process $J(t)$ characterized by the autocorrelation function $\left(\tau=t_{1}-t_{2}\right)$

$$
R_{\mathrm{J}}(\tau)=P_{\mathrm{J}} \frac{\sin \left(\pi B_{\mathrm{J}} \tau\right)}{\pi B_{\mathrm{J}} \tau} \cos \left(2 \pi f_{\mathrm{J}} \tau\right) .
$$

It depends on the mean interference power $P_{\mathrm{J}}$ determined by the ratio of the interferer's bit energy $E_{\mathrm{b}, \mathrm{J}}$ and bit duration $T_{\mathrm{b}, \mathrm{J}}=q T_{\mathrm{b}}, q>0$. Further parameters are the interference center frequency $f_{\mathrm{J}}$ as well as its bandwidth $B_{\mathrm{J}}{ }^{1}$. The resulting interferer's signal duration $T_{\mathrm{p}, \mathrm{J}} \approx \frac{1}{B_{\mathrm{J}}} \leq T_{\mathrm{b}, \mathrm{J}}$ leads to an interference duty cycle of $d_{\mathrm{J}}=\frac{T_{\mathrm{p}, \mathrm{J}}}{T_{\mathrm{b}, \mathrm{J}}}=\frac{d_{\mathrm{s}} T_{\mathrm{p}, \mathrm{J}}}{q T_{\mathrm{p}}}$.

The received signal of (4) is first bandpass filtered and afterwards put into noncoherent energy detection with observation period $T_{\mathrm{p}}$. At its input stage the available SINR is given by

$$
\mathrm{SINR}_{\mathrm{in}}=10 \log _{10} \frac{E_{\mathrm{b}}}{T_{\mathrm{b}}\left(P_{\mathrm{J}}+P_{\mathrm{N}}\right)},
$$

which is identical for OOK/BPPM. In (6) $P_{\mathrm{N}}$ stands for the mean noise power of the passband noise signal, which is modeled as band-limited wide-sense stationary, time-continuous zero mean Gaussian process $N(t):\left(\tau=t_{1}-t_{2}\right)$

$$
R_{\mathrm{N}}(\tau)=P_{\mathrm{N}} \frac{\sin (\pi B \tau)}{\pi B \tau} \cos \left(2 \pi f_{\mathrm{c}} \tau\right) .
$$

At the output of energy detection the decision variable differs for OOK/BPPM.

OOK: For OOK, the asymmetric decision variable

$$
x^{\mathrm{O}}=\int_{0}^{T_{\mathrm{p}}} y^{2}(t) \mathrm{d} t=x_{s}^{\mathrm{O}}+\Delta x^{\mathrm{O}}
$$

occurs. The resulting energy value $x^{\mathrm{O}}$ consists of a deterministic signal-only part

$$
x_{s}^{\mathrm{O}}= \begin{cases}0 & b_{k}=0 \\ 2 E_{\mathrm{b}} & b_{k}=1\end{cases}
$$

\footnotetext{
${ }^{1}$ Eq. (5) holds if the interference source is completely inside the MIR UWB subband. In case $B_{\mathrm{J}}$ overlaps completely or only partially with the subband $f_{\mathrm{J}}, B_{\mathrm{J}}$ and $P_{\mathrm{J}}$ have to be properly modified. However, as it can be ascribed to (5) the following investigations focus solely on an interference source being completely inside the subband.
}

of mean $E_{\mathrm{b}}$ and second order moment $2 E_{\mathrm{b}}^{2}$. The component $\Delta x^{\mathrm{O}}=x_{s j n}^{\mathrm{O}}+x_{j n}^{\mathrm{O}}$ contains the mixed signal-noise and signalinterference term

$$
x_{s j n}^{\mathrm{O}}= \begin{cases}0 & b_{k}=0, \\ 2 \sqrt{\frac{2 E_{\mathrm{p}}^{\mathrm{O}}}{T_{\mathrm{p}}}} \int_{0}^{T_{\mathrm{p}}} \cos \left(2 \pi f_{\mathrm{c}} t\right)(J(t)+N(t)) \mathrm{d} t & b_{k}=1,\end{cases}
$$

as well as the contribution

$$
x_{j n}^{\mathrm{O}}=\int_{0}^{T_{\mathrm{p}}}(J(t)+N(t))^{2} \mathrm{~d} t \quad b_{k}=0,1,
$$

due to noise and interference-only.

BPPM: In contrast to OOK the BPPM decision variable at the output of energy detection is symmetric [8]:

$$
\begin{aligned}
x^{\mathrm{P}} & =\int_{0}^{T_{\mathrm{p}}} y^{2}(t) \mathrm{d} t-\int_{\frac{T_{\mathrm{b}}}{2}}^{\frac{T_{\mathrm{b}}}{2}+T_{\mathrm{p}}} y^{2}(t) \mathrm{d} t \\
& =x_{s}^{\mathrm{P}}+\Delta x^{\mathrm{P}} .
\end{aligned}
$$

The decision variable $x^{\mathrm{P}}$ compares energy values within two observation intervals of duration $T_{\mathrm{p}}$. It is composed of a signalonly contribution

$$
x_{s}^{\mathrm{P}}= \begin{cases}E_{\mathrm{b}} & b_{k}=0, \\ -E_{\mathrm{b}} & b_{k}=1,\end{cases}
$$

which is characterized by mean zero and second order moment $E_{\mathrm{b}}^{2}$. The additional term $\Delta x^{\mathrm{P}}=x_{s j n}^{\mathrm{P}}+x_{j n}^{\mathrm{P}}$ is composed of a mixed signal-noise and signal-interference component

$x_{s j n}^{\mathrm{P}}=\left\{\begin{array}{lr}a \int_{0}^{T_{\mathrm{p}}} \cos \left(2 \pi f_{\mathrm{c}} t\right)(J(t)+N(t)) \mathrm{d} t & b_{k}=0, \\ -a \int_{\frac{T_{\mathrm{b}}}{2}}^{\frac{T_{\mathrm{b}}}{2}+T_{\mathrm{p}}} \cos \left(2 \pi f_{\mathrm{c}}\left(t-\frac{T_{\mathrm{b}}}{2}\right)\right)(J(t)+N(t)) \mathrm{d} t, & b_{k}=1,\end{array}\right.$

with $a=2 \sqrt{2 E_{\mathrm{p}}^{\mathrm{P}} / T_{\mathrm{p}}}$ and the noise and interference-only part

$$
x_{j n}^{\mathrm{P}}=x_{j n}^{\mathrm{O}}-\int_{\frac{T_{\mathrm{b}}}{2}}^{\frac{T_{\mathrm{b}}}{2}+T_{\mathrm{p}}}(J(t)+N(t))^{2} \mathrm{~d} t .
$$

\section{AnAlysis of INTERFERENCE RobUStNeSS}

To make statements on the interference robustness of energy detection for OOK/BPPM a proper quality criterion has to be introduced. A possible measure is the PG of an energy detector. It refers the available SINR at the energy detector's output to the SINR at its input. For OOK this can be described as

$$
\mathrm{PG}^{\mathrm{O}}=10 \log _{10}\left(\frac{2 E_{\mathrm{b}}^{2}}{\frac{1}{2} Q_{1}^{\mathrm{O}}+Q_{2}^{\mathrm{O}}}\right)-10 \log _{10}\left(\mathrm{SINR}_{\mathrm{in}}\right),
$$


which differs from the PG of the BPPM based energy detection receiver expressed as

$$
\mathrm{PG}^{\mathrm{P}}=10 \log _{10}\left(\frac{E_{\mathrm{b}}^{2}}{Q_{1}^{\mathrm{P}}+Q_{2}^{\mathrm{P}}}\right)-10 \log _{10}\left(\mathrm{SINR}_{\mathrm{in}}\right) .
$$

In (16) and (17) $Q_{1}^{i}, i \in\{\mathrm{O}, \mathrm{P}\}$ stands for the second order moment of the mixed signal-noise and signal-interference component $x_{s j n}^{i}, i \in\{\mathrm{O}, \mathrm{P}\}$. In contrast, $Q_{2}^{i}, i \in\{\mathrm{O}, \mathrm{P}\}$ describes the second order moment of the noise and interferenceonly part $x_{j n}^{i}, i \in\{\mathrm{O}, \mathrm{P}\}$.

Based on PG, separate statements on the detection performance can be made for each modulation scheme, i.e., a low modulation specific PG indicates an increased error probability and vice versa. Hence, the smaller $Q_{1}^{i}$ and $Q_{2}^{i}, i \in\{\mathrm{O}, \mathrm{P}\}$ the lower the modulation related error detection probability. In the following, $Q_{1}^{i}$ and $Q_{2}^{i}, i \in\{\mathrm{O}, \mathrm{P}\}$ are determined for both modulation schemes.

OOK: For OOK, the second order moment of the signalnoise and signal-interference part $x_{s j n}^{\mathrm{O}}$ can be formulated as $\left(\tau=t_{1}-t_{2}\right)$

$$
\begin{aligned}
Q_{1}^{\mathrm{O}=} & \frac{8 E_{\mathrm{p}}^{\mathrm{O}}}{T_{\mathrm{p}}} \int_{0}^{T_{\mathrm{p}}} \int_{0}^{T_{\mathrm{p}}} \cos \left(2 \pi f_{\mathrm{c}} t_{1}\right) \cos \left(2 \pi f_{\mathrm{c}} t_{2}\right) \cdot \\
& {\left[E\left(J\left(t_{1}\right) J\left(t_{2}\right)\right)+E\left(N\left(t_{1}\right) N\left(t_{2}\right)\right)\right] \mathrm{d} t_{1} \mathrm{~d} t_{2} } \\
= & \frac{4 E_{\mathrm{p}}^{\mathrm{O}}}{T_{\mathrm{p}}} \int_{0}^{T_{\mathrm{p}}} \int_{0}^{T_{\mathrm{p}}}\left(R_{\mathrm{J}}(\tau)+R_{\mathrm{N}}(\tau)\right) \cdot\left[\cos \left(2 \pi f_{\mathrm{c}}\left(t_{1}-t_{2}\right)\right)\right. \\
& \left.+\cos \left(2 \pi f_{\mathrm{c}}\left(t_{1}+t_{2}\right)\right)\right] \mathrm{d} t_{1} \mathrm{~d} t_{2} .
\end{aligned}
$$

A solution of $Q_{1}^{\mathrm{O}}$ can be found using Parseval's theorem under the assumptions $2\left|f_{\mathrm{c}}+f_{\mathrm{J}}\right| \gg B_{\mathrm{J}}$ and $4 f_{\mathrm{c}} \gg B$. This leads to the closed-form expression

$$
\begin{aligned}
Q_{1}^{\mathrm{O}}= & E_{\mathrm{p}}^{\mathrm{O}} P_{\mathrm{J}} \sum_{n=0}^{\infty}(-1)^{n}\left(2 \pi T_{\mathrm{p}}\right)^{2 n}\left(\frac{r_{2 n+1} \sum_{l=0}^{2 n+1} u_{n, l}}{2 \pi f_{\mathrm{c}}(2 n+1)}\right. \\
- & \left.\frac{2 \pi T_{\mathrm{p}} r_{2 n+2} \sum_{l=0}^{2 n+2} v_{n, l}}{2 \pi f_{\mathrm{c}}(2 n+2)}+\frac{4 T_{\mathrm{p}} r_{2 n+1}}{(2 n+2) !(2 n+1)}\right) \\
+ & \frac{E_{\mathrm{p}}^{\mathrm{O}} P_{\mathrm{N}}}{2 \pi f_{\mathrm{c}}} \sum_{n=0}^{\infty} \frac{(-1)^{n}\left(\pi T_{\mathrm{p}} B\right)^{2 n}}{(2 n+1)}\left(\frac{8 \pi T_{\mathrm{p}} f_{\mathrm{c}}}{(2 n+2) !}+(1+\right. \\
& \left.\left.\cos \left(4 \pi f_{\mathrm{c}} T_{\mathrm{p}}\right)\right) \sum_{l=0}^{2 n+1} w_{n, l}-\sin \left(4 \pi f_{\mathrm{c}} T_{\mathrm{p}}\right) \sum_{l=0}^{2 n+1} z_{n, l}\right),
\end{aligned}
$$

whereas, with $\Delta_{f_{\mathrm{c}, \mathrm{J}}}=f_{\mathrm{c}}-f_{\mathrm{J}}$, the following notations are used:

$$
\begin{aligned}
r_{\nu}= & \frac{1}{B_{\mathrm{J}}}\left(\left(\frac{B_{\mathrm{J}}}{2}+\Delta_{f_{\mathrm{c}, \mathrm{J}}}\right)^{\nu}-\left(-\frac{B_{\mathrm{J}}}{2}+\Delta_{f_{\mathrm{c}, \mathrm{J}}}\right)^{\nu}\right), \\
w_{n, l}= & \frac{\sin \left(4 \pi f_{\mathrm{c}} T_{\mathrm{p}}+\frac{1}{2} l \pi\right)}{(2 n+1-l) !\left(4 \pi f_{\mathrm{c}} T_{\mathrm{p}}\right)^{l}}, \\
u_{n, l}= & w_{n, l}+\frac{(-1)^{l}}{(2 n+1-l) !} \sum_{k=0}^{l} \frac{\sin \left(4 \pi f_{\mathrm{c}} T_{\mathrm{p}}+\frac{1}{2} k \pi\right)}{(l-k) !\left(4 \pi f_{\mathrm{c}} T_{\mathrm{p}}\right)^{k}}, \\
z_{n, l}= & \frac{\cos \left(4 \pi f_{\mathrm{c}} T_{\mathrm{p}}+\frac{1}{2} l \pi\right)}{(2 n+1-l) !\left(4 \pi f_{\mathrm{c}} T_{\mathrm{p}}\right)^{l}}, \\
v_{n, l}= & \frac{z_{n, l}}{(2 n+2-l)} \\
& -\frac{(-1)^{l}}{(2 n+2-l) !} \sum_{k=0}^{l} \frac{\cos \left(4 \pi f_{\mathrm{c}} T_{\mathrm{p}}+\frac{1}{2} k \pi\right)}{(l-k) !\left(4 \pi f_{\mathrm{c}} T_{\mathrm{p}}\right)^{k}} .
\end{aligned}
$$

Eq. (19) reveals the dependency of $Q_{1}^{\mathrm{O}}$ from the system parameters $E_{\mathrm{p}}^{\mathrm{O}}, T_{\mathrm{p}}, f_{\mathrm{c}}, B$ as well as from the interference parameters $P_{\mathrm{J}}, B_{\mathrm{J}}, f_{\mathrm{J}}$. In addition, concerning the special case $B_{\mathrm{J}} \rightarrow 0$, e.g., a cosine tone, $r_{\nu}$ has to be replaced with $r_{\nu}^{m}=\lim _{B_{\mathrm{J}} \rightarrow 0} r_{\nu}=\nu \Delta_{f_{\mathrm{c}, \mathrm{J}}}^{\nu-1}$. Note that this result is consistent to [9] if $P_{\mathrm{N}}=0$.

The second order moment of the noise and interference-only part $x_{j n}^{\mathrm{O}}$ can be described as

$$
\begin{aligned}
Q_{2}^{\mathrm{O}=} & \int_{0}^{T_{\mathrm{p}}} \int_{0}^{T_{\mathrm{p}}}\left[4 E\left(J\left(t_{1}\right) N\left(t_{1}\right) J\left(t_{2}\right) N\left(t_{2}\right)\right)+\right. \\
& 2 E\left(J^{2}\left(t_{1}\right) N^{2}\left(t_{2}\right)\right)+E\left(N^{2}\left(t_{1}\right) N^{2}\left(t_{2}\right)\right)+ \\
& \left.E\left(J^{2}\left(t_{1}\right) J^{2}\left(t_{2}\right)\right)\right] \mathrm{d} t_{1} \mathrm{~d} t_{2} \\
= & \int_{0}^{T_{\mathrm{p}}} \int_{0}^{T_{\mathrm{p}}}\left[\left(P_{\mathrm{N}}+P_{\mathrm{J}}\right)^{2}+2\left(R_{\mathrm{N}}(\tau)+R_{\mathrm{J}}(\tau)\right)^{2}\right] \mathrm{d} t_{1} \mathrm{~d} t_{2},
\end{aligned}
$$

where $\tau=t_{1}-t_{2}$. Thereby, using the theorem of Price [10], (20) can be written in terms of the noise and interference related autocorrelation functions. With Parseval and the assumptions $2 f_{\mathrm{c}} \gg B, 2 f_{\mathrm{J}} \gg B_{\mathrm{J}}$ and $\left|f_{\mathrm{c}}+f_{\mathrm{J}}\right| \gg\left(B_{\mathrm{J}}\right.$ or $\left.\left(B-B_{\mathrm{J}}\right)\right)$ (20) results in

$$
\begin{aligned}
Q_{2}^{\mathrm{O}} & =2 T_{\mathrm{p}}^{2}\left[P_{\mathrm{J}}^{2}+P_{\mathrm{J}} P_{\mathrm{N}}+P_{\mathrm{N}}^{2}\right. \\
& +\sum_{k=1}^{\infty} \frac{(-1)^{k}\left(2 \pi T_{\mathrm{p}}\right)^{2 k}\left(P_{\mathrm{J}}^{2} B_{\mathrm{J}}^{2 k}+P_{\mathrm{N}}^{2} B^{2 k}\right)}{(2 k+1) !(2 k+1)(k+1)} \\
& \left.+\sum_{k=2}^{\infty} \frac{(-1)^{k}\left(2 \pi T_{\mathrm{p}}\right)^{2 k-2}\left(P_{\mathrm{J}}^{2} B_{\mathrm{J}}^{2 k-2}+P_{\mathrm{N}}^{2} B^{2 k-2}\right)}{(k)(2 k) !}\right] \\
& +\frac{2 P_{\mathrm{J}} P_{\mathrm{N}}}{\pi B\left(f_{\mathrm{p}}-f_{\mathrm{m}}\right)} \sum_{k=0}^{\infty} \frac{(-1)^{k}(2 \pi)^{2 k+1} T_{\mathrm{p}}^{2 k+2}}{(2 k+2) !} \\
& \cdot\left(\frac{f_{1, k}}{2 k+2}+\frac{f_{2, k}}{2 k+1}\right) .
\end{aligned}
$$

Thereby, with $f_{\mathrm{p}}=\frac{B}{2}+\frac{B_{\mathrm{J}}}{2}$ and $f_{\mathrm{m}}=\frac{B}{2}-\frac{B_{\mathrm{J}}}{2}, f_{1, k}$ and $f_{2, k}$ 
are defined as:

$$
\begin{aligned}
f_{1, k}= & \left(-f_{\mathrm{m}}-\Delta_{f_{\mathrm{c}, J}}\right)^{2 k+2}-\left(-f_{\mathrm{p}}-\Delta_{f_{\mathrm{c}, J}}\right)^{2 k+2} \\
& +\left(-f_{\mathrm{m}}+\Delta_{f_{\mathrm{c}, J}}\right)^{2 k+2}-\left(-f_{\mathrm{p}}+\Delta_{f_{\mathrm{c}, J}}\right)^{2 k+2} \\
f_{2, k}= & \left(f_{\mathrm{p}}+\Delta_{f_{\mathrm{c}, J}}\right)\left[\left(-f_{\mathrm{m}}-\Delta_{f_{\mathrm{c}, J}}\right)^{2 k+1}-\left(-f_{\mathrm{p}}-\right.\right. \\
& \left.\left.\Delta_{f_{\mathrm{c}, J}}\right)^{2 k+1}\right] \\
+ & \left(f_{\mathrm{p}}-\Delta_{f_{\mathrm{c}, J}}\right)\left[\left(-f_{\mathrm{m}}+\Delta_{f_{\mathrm{c}, J}}\right)^{2 k+1}-\left(-f_{\mathrm{p}}+\right.\right. \\
& \left.\left.\left.\Delta_{f_{\mathrm{c}, J}}\right)^{2 k+1}\right)\right] \\
& +\left(f_{\mathrm{p}}-f_{\mathrm{m}}\right)\left[\left(f_{\mathrm{m}}-\Delta_{f_{\mathrm{c}, J}}\right)^{2 k+1}-\left(-f_{\mathrm{m}}-\right.\right. \\
& \left.\left.\Delta_{f_{\mathrm{c}, \mathrm{J}}}\right)^{2 k+1}\right] .
\end{aligned}
$$

$Q_{2}^{\mathrm{O}}$ is influenced by the system parameters $T_{\mathrm{p}}, f_{\mathrm{c}}, B$ as well as by the interference parameters $P_{\mathrm{J}}, B_{\mathrm{J}}, f_{\mathrm{J}}$. However, in contrast to $Q_{1}^{\mathrm{O}}$ it cannot be reduced via $E_{\mathrm{p}}^{\mathrm{O}}$. Eq. (21) simplifies for $B_{\mathrm{J}} \rightarrow 0$ due to $P_{\mathrm{J}}^{2} B_{\mathrm{J}}^{2 k}=P_{\mathrm{J}}^{2} B_{\mathrm{J}}^{2 k-2}=0, \frac{f_{1, k}}{f_{\mathrm{p}}-f_{\mathrm{m}}}=$ $(2 k+2)\left[\left(-\frac{B}{2}-\Delta f_{\mathrm{c}, \mathrm{J}}\right)^{2 k+1}+\left(-\frac{B}{2}+\Delta f_{\mathrm{c}, \mathrm{J}}\right)^{2 k+1}\right]$ as well as $\frac{f_{2, k}}{f_{\mathrm{p}}-f_{\mathrm{m}}}=(2 k+1)\left(\frac{B}{2}+\Delta f_{\mathrm{c}, \mathrm{J}}\right)\left(-\frac{B}{2}-\Delta f_{\mathrm{c}, \mathrm{J}}\right)^{2 k}$. Assuming $P_{\mathrm{N}}=0$ for this case, (21) equals the result of [9].

BPPM: Considering BPPM the second order moment of the signal-noise and signal-interference part $x_{s j n}^{\mathrm{P}}$ is: $Q_{1}^{\mathrm{P}}=\frac{1}{2} Q_{1}^{\mathrm{O}} \cdot Q_{1}^{\mathrm{P}}$ differs from $Q_{1}^{\mathrm{O}}$ solely in a factor of two which can be ascribed to the reduced modulation specific pulse energy. In contrast to $Q_{1}^{\mathrm{P}}$ there is a significant difference concerning the second order moment of the noise and interference-only part $x_{j n}^{\mathrm{P}}$. With the theorem of Price this can be again generally described in terms of the noise and interference specific autocorrelation functions: $\left(\tau=t_{1}-t_{2}\right)$

$$
\begin{aligned}
Q_{2}^{\mathrm{P}}= & 2 \int_{0}^{T_{\mathrm{p}}} \int_{0}^{T_{\mathrm{p}}}\left[E\left(J^{2}\left(t_{1}\right) J^{2}\left(t_{2}\right)\right)+4 E\left(J\left(t_{1}\right) N\left(t_{1}\right) .\right.\right. \\
& \left.\left.J\left(t_{2}\right) N\left(t_{2}\right)\right)+E\left(N^{2}\left(t_{1}\right) N^{2}\left(t_{2}\right)\right)\right] \mathrm{d} t_{1} \mathrm{~d} t_{2} \\
& -2 \int_{0}^{T_{\mathrm{p}}} \int_{T_{\mathrm{b}}}^{T_{\mathrm{p}}+\frac{T_{\mathrm{b}}}{2}}\left[E\left(J^{2}\left(t_{1}\right) J^{2}\left(t_{2}\right)\right)+4 E\left(J\left(t_{1}\right) N\left(t_{1}\right) .\right.\right. \\
& \left.\left.J\left(t_{2}\right) N\left(t_{2}\right)\right)+E\left(N^{2}\left(t_{1}\right) N^{2}\left(t_{2}\right)\right)\right] \mathrm{d} t_{1} \mathrm{~d} t_{2} \\
& -4 \int_{0}^{T_{\mathrm{p}}} \int_{0}^{T_{\mathrm{p}}}\left[R_{\mathrm{I}}^{2}(\tau)+R_{\mathrm{N}}^{2}(\tau)+R_{\mathrm{I}}(\tau) R_{\mathrm{N}}(\tau)\right] \mathrm{d} t_{1} \mathrm{~d} t_{2} \\
& 4 \int_{0}^{T_{\mathrm{p}}} \int_{T_{\mathrm{p}}+\frac{T_{\mathrm{b}}}{2}}^{\frac{T_{\mathrm{b}}}{2}}\left[R_{\mathrm{I}}^{2}(\tau)+R_{\mathrm{N}}^{2}(\tau)+R_{\mathrm{I}}(\tau) R_{\mathrm{N}}(\tau)\right] \mathrm{d} t_{1} \mathrm{~d} t_{2} .
\end{aligned}
$$

Therefore, using the theorem of Parseval for $2 f_{\mathrm{J}} \gg B_{\mathrm{J}}$, the closed-form result

$$
\begin{aligned}
Q_{2}^{\mathrm{P}} & =2 \sum_{k=1}^{\infty} \frac{(-1)^{k}(2 \pi)^{2 k}\left(P_{\mathrm{J}}^{2} B_{\mathrm{J}}^{2 k}+P_{\mathrm{N}}^{2} B^{2 k}\right) g_{2 k+2}}{(2 k+1) !(2 k+1)(k+1)} \\
& +\sum_{k=2}^{\infty} \frac{(-1)^{k} 2^{2 k}(\pi)^{2 k-2}\left(P_{\mathrm{J}}^{2} B_{\mathrm{J}}^{2 k-2}+P_{\mathrm{N}}^{2} B^{2 k-2}\right) g_{2 k}}{(2 k) !(2 k)} \\
& +\frac{2 P_{\mathrm{J}} P_{\mathrm{N}}}{\pi B\left(f_{\mathrm{p}}-f_{\mathrm{m}}\right)} \sum_{k=0}^{\infty} \frac{(-1)^{k}(2 \pi)^{2 k+1} g_{2 k+2}}{(2 k+2) !} \\
& \cdot\left(\frac{f_{1, k}}{2 k+2}+\frac{f_{2, k}}{2 k+1}\right),
\end{aligned}
$$

with

$$
g_{\nu}=2 T_{\mathrm{p}}^{\nu}-\left(T_{\mathrm{p}}-\frac{T_{\mathrm{b}}}{2}\right)^{\nu}+2\left(\frac{T_{\mathrm{b}}}{2}\right)^{\nu}-\left(T_{\mathrm{p}}+\frac{T_{\mathrm{b}}}{2}\right)^{\nu},
$$

can be found. $Q_{2}^{\mathrm{P}}$ is influenced by the system parameters $T_{\mathrm{p}}$, $T_{\mathrm{b}}, f_{\mathrm{c}}, B$ as well as on the interference parameters $P_{\mathrm{J}}, B_{\mathrm{J}}, f_{\mathrm{J}}$. Similar to $Q_{2}^{\mathrm{O}}$ it cannot be reduced via $E_{\mathrm{p}}^{\mathrm{P}}$. For $B_{\mathrm{J}} \rightarrow 0$, (23) allows the same simplifications as for $Q_{2}^{\mathrm{O}}$. In addition, (23) reveals that for low data rates $\left(T_{\mathrm{b}} \rightarrow \infty\right) g_{\nu} \approx 2 T_{\mathrm{p}}^{\nu}$ resulting in a negligible influence of $Q_{2}^{\mathrm{P}}$. In contrast, the larger the data rate the higher its impact, e.g., for the maximum data rate of $T_{\mathrm{b}}=2 T_{\mathrm{p}} g_{\nu}$ conducts to $g_{\nu}=4 T_{\mathrm{p}}^{\nu}-\left(2 T_{\mathrm{p}}\right)^{\nu}$.

\section{RESUlTS}

Based on the previous analysis this section identifies the interference robustness of an OOK/BPPM based energy detection receiver. Thereby, assuming regulation of ECC [2] an MIR UWB system with four subbands of equal bandwidth $B=625 \mathrm{MHz}$ is taken into account. Without loss of generality, the analysis focuses solely on the first subband located at $f_{\mathrm{c}}=6.3125 \mathrm{GHz}$. However, an extension to other subbands or other MIR UWB system configurations, which are possibly based on other frequency masks, e.g., FCC [1], is easily possible. Further common system parameters used in the subsequent analysis are the pulse duration $T_{\mathrm{p}}=3.2 \mathrm{~ns}$, a duty cycle $d_{\mathrm{s}}=\frac{1}{2}$, a mean transmit power normalized to one, the modulation specific pulse energy $E_{\mathrm{p}}^{i}, i \in\{\mathrm{O}, \mathrm{P}\}$ as well as a constant signal-to-noise ratio (SNR) of $10 \mathrm{~dB}$ at the input of the energy detector. Fixed interference parameter is the interference bit duration $T_{\mathrm{b}, \mathrm{J}}=16 T_{\mathrm{b}}=102.4 \mathrm{~ns}$.

In Fig. 1 the PG is plotted vs. the $\mathrm{SINR}_{\text {in. }}$ An interference source with the two bandwidths $B_{\mathrm{J}, 1}=20 \mathrm{MHz}$ and $B_{\mathrm{J}, 2}=400 \mathrm{MHz}$ is considered leading to the fixed duty cycles $d_{\mathrm{J}, 1}=\frac{1}{B_{\mathrm{J}, 1} T_{\mathrm{b}, \mathrm{J}}}=0.4883$ and $d_{\mathrm{J}, 2}=\frac{1}{B_{\mathrm{J}, 2} T_{\mathrm{b}, \mathrm{J}}}=0.0244$. For OOK/BPPM, the PG increases with higher SINR $\mathrm{Sin}_{\text {in }}$ up the interference-free PG at SINR $=10 \mathrm{~dB}$. Furthermore, it can be observed that the OOK/BPPM based PG varies with the interference bandwidth. For OOK, the PG increases with a larger interference bandwidth because of the minor impact of the mixed signal-interference as well as the interference-only component involved in the energy detection. A PG of energy detection can be achieved from a SINR $\operatorname{Sin}_{1}=-3.5 \mathrm{~dB}\left(B_{\mathrm{J}, 1}=\right.$ $20 \mathrm{MHz})$ and from $\mathrm{SINR}_{\mathrm{in}}=-5.5 \mathrm{~dB}\left(B_{\mathrm{J}, 2}=400 \mathrm{MHz}\right)$, respectively. For strong narrow- and broadband interference 


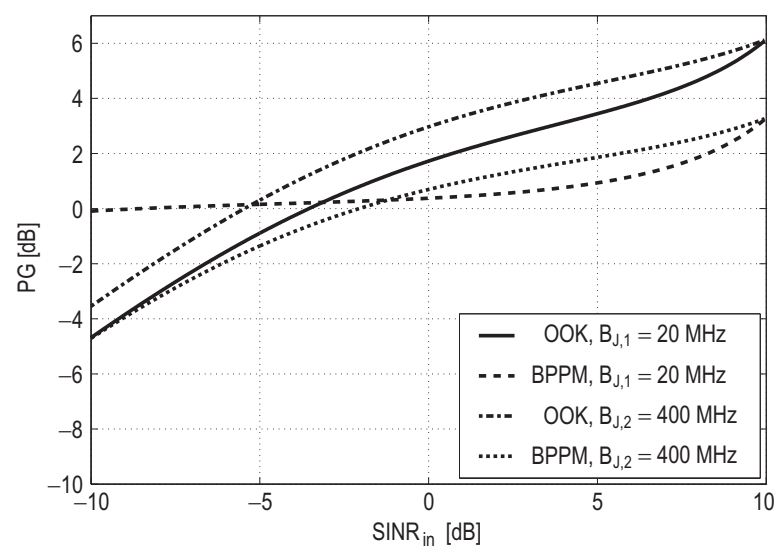

Fig. 1. PG of OOK/BPPM vs. $\mathrm{SINR}_{\text {in }}$ for $B_{\mathrm{J}, 1}=20 \mathrm{MHz}$ and $B_{\mathrm{J}, 2}=$ $400 \mathrm{MHz}$ with $f_{\mathrm{J}}=f_{\mathrm{c}}+50 \mathrm{MHz}, \mathrm{SNR}=10 \mathrm{~dB}, d_{\mathrm{J}}\left(B_{\mathrm{J}, 1}=20 \mathrm{MHz}\right)=$ $0.4883, d_{\mathrm{J}}\left(B_{\mathrm{J}, 2}=400 \mathrm{MHz}\right)=0.0244$ and $T_{\mathrm{b}, \mathrm{J}}=102.4 \mathrm{~ns}$.

no PG results as the energy detector's decision variable (8) is significantly corrupted. In contrast, considering BPPM a PG can be achieved for small interference bandwidths, e.g., $B_{\mathrm{J}, 1}=20 \mathrm{MHz}$, over nearly the complete $\mathrm{SINR}_{\mathrm{in}}$ range. For $B_{\mathrm{J}, 2}=400 \mathrm{MHz}$ a PG occurs from SINR in $=-2 \mathrm{~dB}$. The reason for this behavior lies in a different amount of energy resulting from the mixed signal-interference and interferenceonly term within the two observation periods of duration $T_{\mathrm{p}}$ (12). Finally, considering OOK/BPPM with respect to their relative PG shows that for strong NBI BPPM is more robust whereas $\mathrm{OOK}$ is more robust for mean and low interference.

Fig. 2 shows the PG vs. $f_{\mathrm{J}}$, which varies from $f_{\mathrm{c}}-\frac{B}{2}$ to

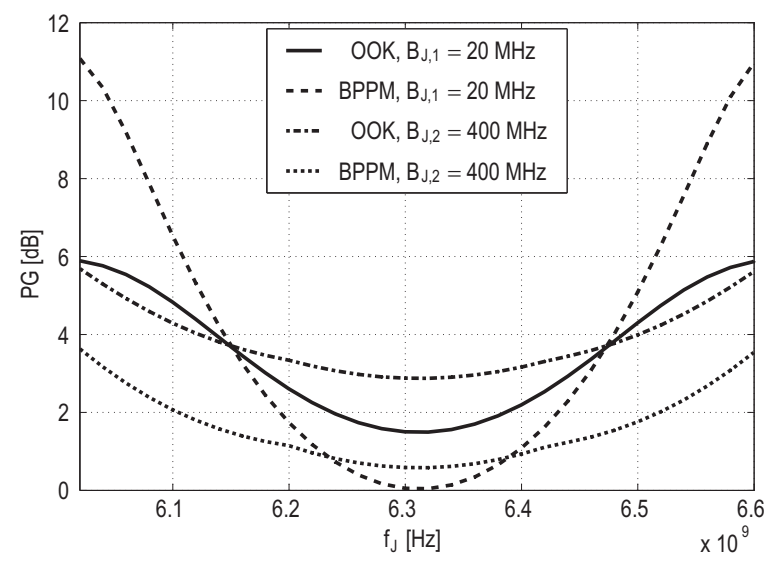

Fig. 2. PG of OOK/BPPM vs. $f_{\mathrm{J}}$ for $\mathrm{SINR}_{\mathrm{in}}=0 \mathrm{~dB}, \mathrm{SNR}=10 \mathrm{~dB}$, $B_{\mathrm{J}, 1}=20 \mathrm{MHz}, B_{\mathrm{J}, 2}=400 \mathrm{MHz}$ and $T_{\mathrm{b}, \mathrm{J}}=102.4 \mathrm{~ns}$.

$f_{\mathrm{c}}+\frac{B}{2}$ for a fixed $\mathrm{SINR}_{\mathrm{in}}=0 \mathrm{~dB}, \mathrm{SNR}_{\mathrm{in}}=10 \mathrm{~dB}, T_{\mathrm{b}, \mathrm{J}}=$ $102.4 \mathrm{~ns}$ as well as for $B_{\mathrm{J}, 1}=20 \mathrm{MHz}$ and $B_{\mathrm{J}, 2}=400 \mathrm{MHz}$. As long as interference is completely inside the subband $T_{\mathrm{p}, \mathrm{J}}$, $d_{\mathrm{J}}$ and hence $P_{\mathrm{J}}$ are fix. In particular, the PG of OOK/BPPM at $f_{\mathrm{J}}=f_{\mathrm{c}}+50 \mathrm{MHz}$ coincides with the one of Fig. 1 . In addition, both modulation schemes show an increase of PG the more the interference source is located at the subband's boundary
$\left(f_{\mathrm{J}}=f_{\mathrm{c}} \pm \frac{1}{T_{\mathrm{p}}}\right)$. This can be on one hand ascribed to the subband pulse's sinc spectrum which is zero at the subband's boundary. On the other hand, the more $f_{\mathrm{J}}$ is located at the subband's boundary the minor the interference bandwidth falling into the subband. In case interference overlaps with the subband's boundary, the effective interference parameters $B_{\mathrm{J}}, f_{\mathrm{J}}$ and $d_{\mathrm{J}}$ changes resulting in a reduction of the actual mean interference power $P_{\mathrm{J}}$.

\section{CONClusion}

This paper investigates the energy detector's robustness in presence of narrow- and broadband interference within an OOK/BPPM based noncoherent MIR UWB communication system. Based on the energy detector's PG closed-form expressions of noise and interference related second order moment statistics at the output of an energy detection receiver are provided. This reveals insight into the impact of interference and system specific parameters. Furthermore, the analysis of the relative modulation specific PG with respect to various interference parameters shows the robustness of OOK/BPPM.

Future work focuses on the approach's extension to other pulse shapes, e.g., cosine-shaped pulses, to other modulation schemes as well as to realistic channel models.

\section{ACKNOWLEDGMENT}

The authors gratefully acknowledge that their work is partially supported within the priority program No. 1202/2 (UKoLoS) by the German Research Foundation (DFG).

\section{REFERENCES}

[1] Federal Communications Commission, "Revision of part 15 of the commission's rules regarding ultra-wideband transmission systems," First Report and Order, ET Docket 98-153, FCC 02-28, adopted/released Feb. 23, 2004/Apr. 22, 2002.

[2] Electronic Communications Commission, "ECC decision of 24 march 2006 on the harmonised conditions for devices using ultra-wideband (UWB) technology in bands below $10.6 \mathrm{GHz}$," Tech. Rep.

[3] K. Witrisal, G. Leus, G. Janssen, M. Pausini, F. Troesch, T. Zasowski, and J. Romme, "Noncoherent Ultra-Wideband Systems," IEEE Signal Process. Mag., vol. 26, no. 4, pp. 48-66, July 2009.

[4] M. Hauske, H. Jäkel, H. Dehner, and F. K. Jondral, "Interference mitigation for energy detection in an multiband impulse radio UWB system," in Proc. of IEEE VTC Fall, Calgary, Canada, pp. 1-5, Sept. 2008.

[5] H. Dehner, A. Romero, H. Jäkel, D. Burgkhardt, R. Moorfeld, F. K. Jondral, and A. Finger, "Iterative coexistence approaches for noncoherent multi-band impulse radio UWB," in Proc. IEEE Int. Conf. Ultra-Wideband, Vancouver, Canada, pp. 734-738, Sept. 2009.

[6] H. Dehner, H. Jäkel, D. Burgkhardt, F. K. Jondral, R. Moorfeld, and A. Finger, "Treatment of Temporary Narrowband Interference in Noncoherent Multiband Impulse Radio UWB," in Proc. IEEE Mediterranean Electrotechnical Conference, Valletta, Malta, pp. 1335-1339, Apr. 2010.

[7] H. Dehner, H. Jäkel, D. Burgkhardt, and F. K. Jondral, "The TeagerKaiser Energy Operator in Presence of Multiple Narrowband Interference," IEEE Communications Letters, vol. 14, no. 8, pp. 716-718, Aug. 2010.

[8] C. Steiner and A. Wittneben, "On the interference robustness of ultrawideband energy detection receivers," in Proc. IEEE Int. Conf. UltraWideband, Singapore, pp. 721-726, Sept. 2007.

[9] H. Dehner, Y. Koch, H. Jäkel, D. Burgkhardt, F. K. Jondral, R. Moorfeld, and A. Finger, "Narrow-band Interference Robustness for Energy Detection in OOK/PPM," in Proc. IEEE Int. Conf. Commun., Cape Town, South Africa, pp. 1-5, May 2010.

[10] A. Papoulis, Ed., Probability, Random Variables, and Stochastic Processes. McGraw-Hill Series in Systems Science, 1965. 